\title{
Optically induced potential energy landscapes
}

\author{
Justo Rodríguez, Luciana C. Dávila Romero, and David L. Andrews
}

School of Chemical Sciences, University of East Anglia, Norwich NR4 7TJ, U.K.

\begin{abstract}
Multi-dimensional potential energy surfaces are associated with optical binding. A detailed exploration of the available degrees of geometric freedom reveals unexpected turning points, producing intricate patterns of local force and torque. Although optical pair interactions outweigh Casimir-Polder coupling even over short distances, the forces are not always attractive. Numerous local potential minimum and maximum can be located, and mapped on contour diagrams. Islands of stability appear, and structures conducive to the formation of rings. The results, based on quantum electrodynamics, apply to optically trapped molecules, nanoparticles, microparticles and colloids.
\end{abstract}

Keywords: Optical binding, optical matter, quantum electrodynamics, optical manipulation, nano-manipulation

Electrically neutral molecules and larger nanoparticles, separated from each other beyond significant wavefunction overlap, generally experience weak forces of mutual attraction. Typically, such forces are associated with pair potentials characterized by an inverse sixth power dependence on inter-particle distance. In the case of molecules, the latter feature (the attractive component of a Lennard-Jones interaction) is interpreted as a dynamic coupling of fluctuating electric dipoles. Experimentally verifiable retardation effects modify the distance dependence at longer distances, exhibiting the deeply quantum electrodynamical character of the Casimir-Polder interaction [1-3] - ultimately attributable to virtual photon exchange. The additive effect of pairwise interactions between the constituents of larger particles conveys an inverse sixth power distance relationship into the Hamaker interaction [4], where it determines properties such as adhesion, wetting, multilayer adsorption and colloid flocculation [5,6].

A suspicion that intense laser light might engage with virtual photon exchange, producing an optically modified potential energy surface, was first developed into theory by Thirunamachandran almost thirty years ago [7] - but the laser intensities that appeared necessary then represented a major deterrent. Within the decade, however, a landmark paper by Burns et al. [8] verified the effect experimentally, and introduced the term 'optical binding'; this work also drew attention to the long-range linearly inverse dependence on distance, tempered by an oscillatory factor. The study inspired increasingly adventurous theoretical and experimental investigations [9-22] and the development of quantum electrodynamical studies $[23,24]$. Mostly, attention has focused on the intensity and distance dependence of the pair forces. The pair separation is, however, only one of the geometric degrees of freedom. In this Letter, we present the first results to emerge from an investigation into the complete potential energy surfaces for optically induced interactions. Despite a relative simplicity in the field equations, a richly textured potential energy landscape emerges.

For a pair of particles, B displaced from A by a vector $\boldsymbol{R}$, each with a dynamic polarizability tensor $\alpha(-k ; k)$, the optical potential is concisely expressible as follows [24,25];

$$
\begin{aligned}
\Delta E(\mathbf{k}, \mathbf{R}) & =\left(\frac{k^{3} I(c k)}{4 \pi c \varepsilon_{0}^{2}}\right) \operatorname{Img} g^{(1)}(\mathbf{R})\left[\delta_{j k}\left\{h_{0}^{(1)}(k R)-k^{-1} R^{-1} h_{1}^{(1)}(k R)\right\}+\hat{R}_{j} \hat{R}_{k} h_{2}^{(1)}(k R)\right] e_{i}^{(\lambda)} \bar{e}_{l}^{(\lambda)} \\
& \times\left[\alpha_{i j}^{\mathrm{A}}(-k ; k) \alpha_{k l}^{\mathrm{B}}(-k ; k) \exp (\mathbf{i k . R})+\alpha_{i j}^{\mathrm{B}}(-k ; k) \alpha_{k l}^{\mathrm{A}}(-k ; k) \exp (-\mathrm{ik} . \mathbf{R})\right] .
\end{aligned}
$$


Here, $\boldsymbol{k}$ is the wave-vector of the throughput radiation and $k=|\boldsymbol{k}| ; \boldsymbol{e}^{(\lambda)}$ is the (complex) polarization vector, $I$ the mean irradiance and $g^{(1)}(\mathbf{R}) \equiv g^{(1)}\left(r_{\mathrm{A}} ; r_{\mathrm{B}}\right)$ the time-averaged degree of first-order coherence (correlating the positive frequency signal at A with its negative frequency counterpart at $\mathrm{B}) ; h_{n}^{(1)}(z)=(\pi / 2 z)^{1 / 2} H_{n+(1 / 2)}^{(1)}(z)$ are spherical Hankel functions of the first kind; $c$ is the speed of light in vacuum, $\varepsilon_{0}$ the permittivity in vacuum, and $\delta_{j k}$ the Kronecker delta. Generally the coherence length of the radiation exceeds the pair separation, and the degree of coherence can be taken as unity. In applying (1), it is assumed that the particle pair is held by an optical trap or suspension that exercises an equal force on each particle, so the inter-particle potential is entirely separable.

Even the simplest two-particle case has at least three degrees of geometric freedom. As is apparent from (1), the interaction engages the directions of both the optical propagation and polarization vectors; the plane containing these two, together with its normal, establishes a Cartesian frame against which the pair orientation may be defined. When the particles are spherical, there are no further degrees of freedom for the orientations of the individual particles, and for each pair an obvious choice of coordinates is the pair separation $R$ and two angles, $\phi$ and $\zeta$ specifying the pair orientation - see Fig. 1. In terms of these variables the solution (1) substantially simplifies as follows:

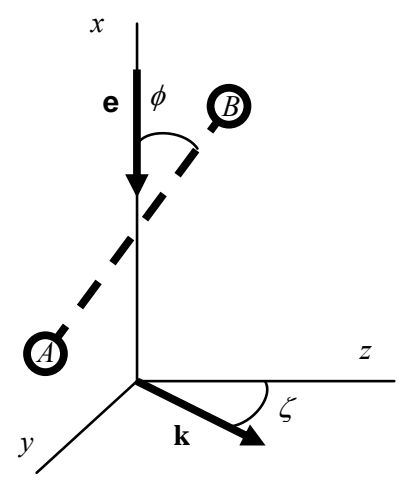

Fig. 1. Two-particle geometry. Trapped particles $\mathrm{A}$ and $\mathrm{B}$, separated by distance $R$, their inter-particle displacement vector subtending an angle $\phi$ to the electric field of the laser radiation, and $\zeta$ designating the angle between the plane containing these vectors and the optical wave-vector.

$$
\begin{aligned}
\Delta E=\left(\frac{I(c k)}{2 \pi c \varepsilon_{0}^{2} R^{3}}\right) & \mathcal{R e}\left[\alpha^{2}(-k ; k) \cos (k R \sin \phi \cos \varsigma)\right. \\
& \times\left\{\left((1-\mathrm{i} k R)\left(1-3 \cos ^{2} \phi\right)-k^{2} R^{2} \sin ^{2} \phi\right\} \exp (\mathrm{i} k R)\right],
\end{aligned}
$$

where the polarization is taken as linear and $\alpha$ is the scalar polarizability. As the distance between the pair increases, the optically induced force rapidly overtakes in magnitude the more sharply distance-dependent Casimir force. Over short distances a convenient basis for comparison with the radiation-free Casimir-Polder energy of interaction is the dimensionless parameter $\hbar c^{2} k / I R^{3}\left(\hbar c^{2} k^{3} / I R^{5}\right.$ in the long-range, $\left.k R » 1\right)$. With a wavelength of $355 \mathrm{~nm}$ and a modest irradiance of $10^{12} \mathrm{~W} \mathrm{~m}^{-2}$, the optically induced interaction typically exceeds the Casimir-Polder force at all distances beyond about $55 \mathrm{~nm}$, subject to the pair orientation.

When the pair of particles is aligned with the polarization vector, equation (2) delivers a result whose graph exhibits the familiar, experimentally verified $R$-dependence [26]. This 
result (alone) is independent of $\zeta$. For the angle dependence in general, it is a trivial matter to prove that, in the near-zone, the isotropic average of the optically induced pair energy vanishes. Nonetheless at all distances the energy landscape is decidedly anisotropic, and must therefore generate both positive and negative forces. Indeed, it has been commented [24] that the term 'optical binding' is potentially misleading, focusing only on attraction forces. Force fields are derivable from (2), but they can also be inferred from the potential energy landscape, since the pictorial representation of a scalar field is also visually less cluttered than a scalar field, we graph the energies. Results from typical calculations are shown in Figs. 2 \& 3.

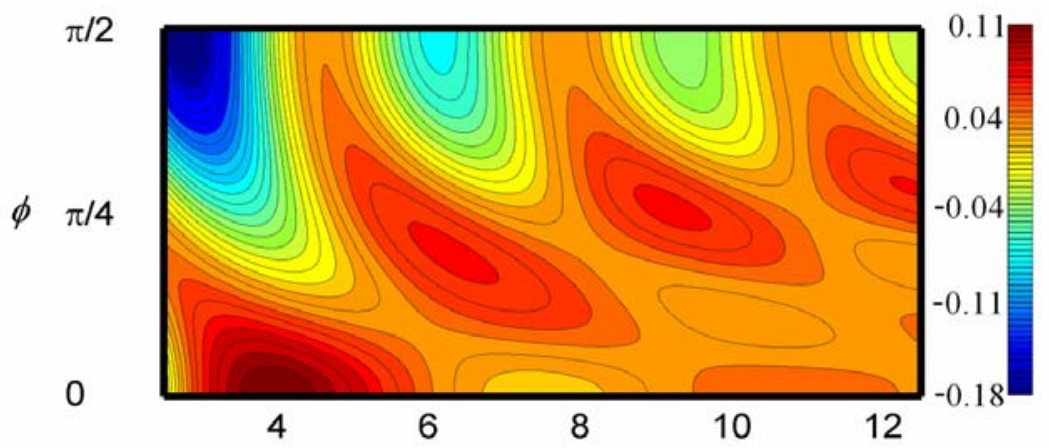

(a) $\quad k R$

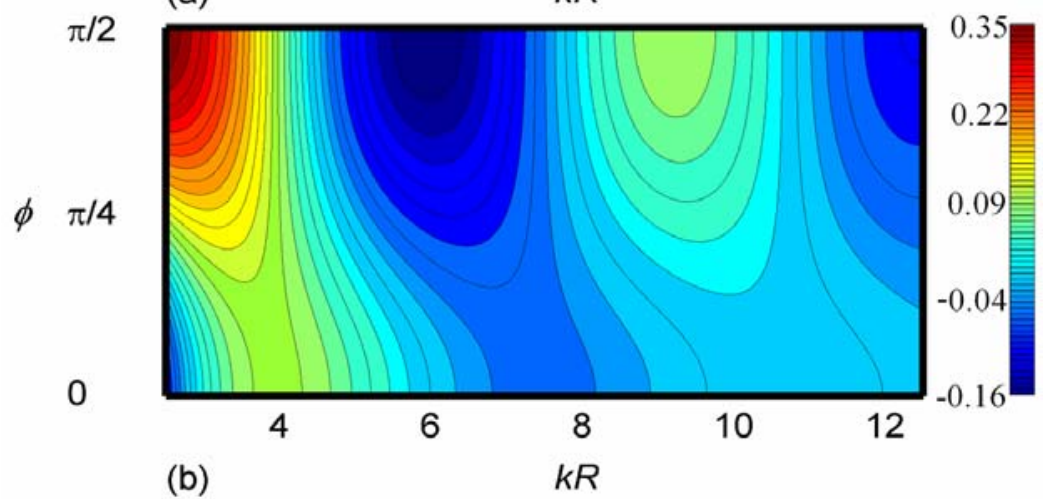

Fig. 2. Contour maps of optically induced pair energy. Plots of $\Delta E$ as a function of $\phi$ and $k R$ : (a) $\zeta=0$; (b) $\zeta=\pi / 2$. The variation of $\Delta E$ with $k R$ along the abscissa, $\phi=0$, shows its first two maxima at $k R \sim 4.0,10.5$, the first (non-proximal) minimum, at

$k R \sim 7.5$. The horizontal scale typically spans distances $R$ of several hundred nanometers, depending on the value of $k$ (see text). The units of the color scale are

$$
2 I k^{3} \alpha^{2}(-k ; k) /\left(4 \pi \varepsilon_{o}^{2} c\right) \text {. }
$$

A host of interesting features emerge even from the handful of examples shown here (a more extensive set being available as supplementary material from the authors). In each landscape, local minima distinguish optical binding configurations. The contours intersect the abscissa scale orthogonally, reflecting an even dependence on each angular variable; the variation in the domain $\tilde{(} \pi / 2, \pi / 2)$ is notionally revealed by unfolding along the distance axis. The physical significance is that a system whose $(k R, \zeta, \phi)$ configuration has $\phi=0$ or $\zeta=0$, but not situated at a local minimum, is always subject to a force drawing it towards a neighboring minimum without change of orientation. For the same reason, there is no torque when $\phi=\pi / 2$ or $\zeta=\pi / 2$. Clearly, however, not all routes to the closest minimum can involve 
a change in only one variable; a system in an arbitrary configuration will generally be subject to forces leading to both forces and torques. For example, inspection of Fig. 2(a) shows that whilst a pair in the configuration $(6.0, \pi / 4,0)$ is subject to a torque tending to increase $\phi$. to $\pi / 2$, its trajectory will be accompanied by forces that tend to first increase and then decrease $R$. The details, which will additionally involve changes in $\zeta$, can of course be determined from the total derivative of (2). Other features, also exemplified in Fig. 2(a), are off-axis islands of stability such as the one that can be identified at $(10, \pi / 10,0)$. The two maps shown in Fig. 3, plotted in the $k R$ range $(2,50)$, illustrate the emergence of potential energy features supporting longer-range order. Notably, columnar patterns of alternating minima and maxima at constant $k R$ represent conditions conducive to the formation of ring structures.
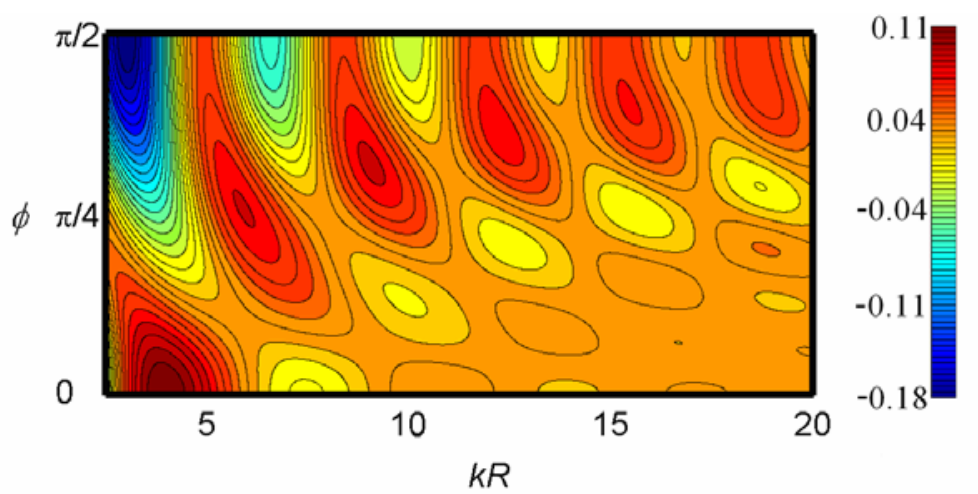

(a)

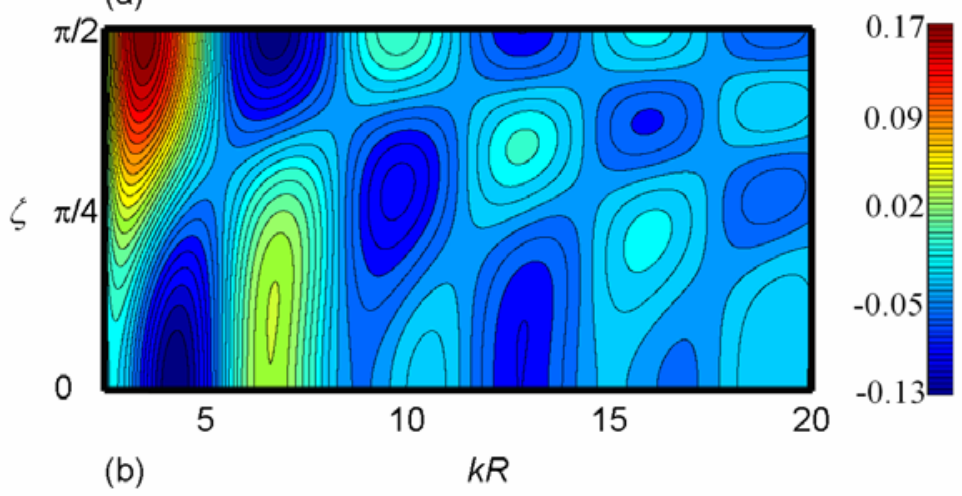

Fig. 3. Contour maps of optically induced pair energy. (a) $\Delta E$ plotted against $\phi$ and $k R ; \zeta=0$, as in Fig. 2(a) on expanded distance scale. (b) $\Delta E$ variation with $\zeta$ and $k R$, at constant $\phi=\pi / 4$. Adjacent, differently colored islands signify alternating local maxima and minima. The $\phi=\pi / 4$ section of the landscape in (a) corresponds to the $\zeta=0$ section in (b). The units of the color scale are $2 I k^{3} \alpha^{2}(-k ; k) /\left(4 \pi \varepsilon_{o}{ }^{2} c\right)$.

The pattern of variation in the pair potential energy, as a function of each of its degrees of freedom, is most readily comprehended in a video visualization. In the example shown here, the variations with $\phi$ and $k R$ are displayed as functions of $\zeta(0 \leq \zeta \leq \pi)$. The most striking features are the tidal ripples that signify the outward (increasing $R$ ) displacements of the maxima and minima up to $\zeta=\pi / 2$, followed by inward displacements over the range $(\pi / 2, \pi)$. For any particle pair trapped in a local minimum, the result signifies a physical effect in which 


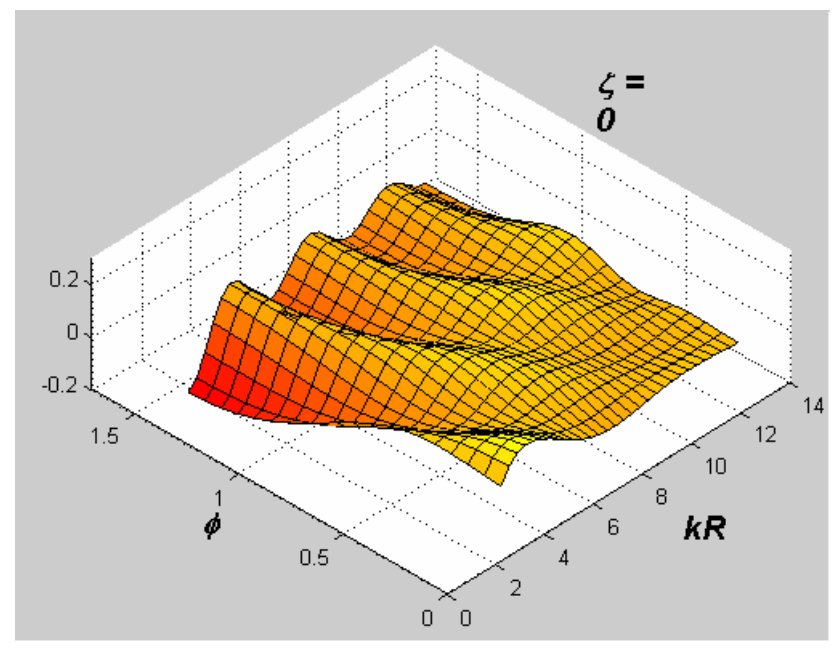

Video 1 . Variation of optically induced pair energy with $\zeta(0 \leq \zeta \leq \pi)$. The units on the $\mathrm{z}$ axes are $2 I k^{3} \alpha^{2}(-k ; k) /\left(4 \pi \varepsilon_{o}{ }^{2} c\right)$ (MPEG, $\left.1446 \mathrm{~KB}\right)$.

pair rotation produces alternately repulsive and attractive forces, manifest as an oscillation in separation distance. The period of that oscillation is half the rotation time.

Our results represent the first fruits of a project to map out the force fields for a large number of interacting particles. The utilization of pair potentials in determining the behavior of an ensemble should not itself be problematic, as the systems of interest are dominated by nearest-neighbor influence, and three-centre interactions are insignificant. Indeed, others have already shown that some calculational complexity can be offset by translational symmetry constraints [17]. The registration of local field effects (for example for microparticles suspended in a liquid) is also straightforward; it is known how the retarded potential essentially the term in the first square brackets in (1) - is affected [27]. Alongside the inclusion of Lorentz field factors, the dependence on $k R$ changes to a dependence on $n(c k) k R$, the multiplier being the refractive index. For example in a liquid illuminated by $355 \mathrm{~nm}$ radiation, with a refractive index at that wavelength of 1.40 , a potential energy minimum registered in the above graphs at $k R \sim 4$ signifies a closer pair separation of $160 \mathrm{~nm}$, rather than $225 \mathrm{~nm}$.

Substantially greater complexity is to be found in systems comprising non-spherical particles, where the number of degrees of freedom rises dramatically. One case of intermediate complexity, that of two cylindrical particles with seven geometric degrees of freedom, has been the focus of some preliminary work, [28] being applied specifically to carbon nanotubes. The theory that furnishes equation (1) is nonetheless general, and capable of delivering detailed results for even this scenario; this is the subject of ongoing work. The results presented here, relating to spherical particles, nonetheless apply to almost all of the systems that have thus far been the focus of experimental studies.

Acknowledgement. We are pleased to acknowledge EPSRC funding.

\section{REFERENCES}

[1] P. W. Milonni and M. L. Shih, "Source theory of the Casimir force," Phys. Rev. A 45, 4241-4253 (1992).

[2] E.A. Power and T. Thirunamachandran, "Casimir potentials: a chemical viewpoint," $J$. Mol. Struct. - Theochem 591, 19-25 (2002). 
[3] F. Capasso, J. N. Munday, D. Iannuzzi, and H. B. Chan, "Casimir forces and quantum electrodynamical torques: Physics and nanomechanics". IEEE J. Sel. Top. Quantum Electron. 13, 400-414 (2007).

[4] R. F. Rajter, R. H. French, W. Y. Ching, W. C. Carter, and Y. M. Chiang, "Calculating van der Waals-London dispersion spectra and Hamaker coefficients of carbon nanotubes in water from ab initio optical properties," J. Appl. Phys. 101, 054303 (2007).

[5] J. Laven and J. P. C. Vissers, "The Hamaker and the Lifshitz approaches for the Van der Waals interaction between particles of composite materials dispersed in a medium," Coll. Surf. A. 152, 345-355 (1999).

[6] H. Wennerstrom, "The van der Waals interaction between colloidal particles and its molecular interpretation," Coll. Surf. A. 228, 189-195 (2003).

[7] T. Thirunamachandran, "Intermolecular interactions in the presence of an intense radiation-field," Mol. Phys. 40, 393-399 (1980).

[8] M. M. Burns, J.-M. Fournier, and J.A. Golovchenko, "Optical binding," Phys. Rev. Lett. 63, 1233-1236 (1989).

[9] F. Depasse and J.-M. Vigoureux, "Optical binding force between two Rayleigh particles," J. Phys. D: Appl. Phys. 27, 914-919 (1994).

[10] M. Nieto-Vesperinas, P. C. Chaumet, and A. Rahmani, "Near-field photonic forces." Phil. Trans. R. Soc. Lond, A 362, 719-737 (2004).

[11] S. K. Mohanty, J. T. Andrews, and P. K. Gupta, "Optical binding between dielectric particles," Opt. Express 12, 2746-2753 (2004).

[12] J.-M. Fournier, J. Rohner, P. Jacquot, R. Johann, S. Mias, and R.-P. Salathé, "Assembling mesoscopic particles by various optical schemes," in Optical Trapping and Optical Micro-manipulation II, ed. K. Dholakia \& G. C. Spalding, Proc. SPIE 5930, $59300 Y$ (2005).

[13]D. L. Andrews, R. G.Crisp, and D. S. Bradshaw, "Optically induced inter-particle forces: from the bonding of dimers to optical electrostriction in molecular solids," J. Phys. B. 39, S637-S650 (2006).

[14] C. D. Mellor, T. A. Fennerty, and C. D. Bain, "Polarization effects in optically bound particle arrays," Opt. Express 14, 10079-10088 (2006).

[15]D. Maystre and P. Vincent, "Making photonic crystals using trapping and binding optical forces on particles," J. Opt. A: Pure Appl. Opt. 8, 1059-1066 (2006).

[16] T. M. Grzegorczyk, B. A. Kemp, and J. A. Kong, "Passive guiding and sorting of small particles with optical binding forces," Opt. Lett. 31, 3378-3380 (2006).

[17] V. Karásek, K. Dholakia, and P. Zemánek, "Analysis of optical binding in one dimension," Appl. Phys. B 84, 149-156 (2006).

[18] T. M. Grzegorczyk, B. A. Kemp, and J. A. Kong, "Stable optical trapping based on optical binding forces," Phys. Rev. Lett. 96, 113903 (2006).

[19] R. G. Crisp and D. L. Andrews, "Optical electrostriction," in Complex Light and Optical Forces, ed. D. L. Andrews, E. J. Galvez \& G. Nienhuis, Proc. SPIE 6483, 648304 (2007).

[20]F. Chen, G. L. Klimchitskaya, V. M. Mostapanenko, and U. Mohideen, "Demonstration of optically modulated dispersion forces," Opt. Express 15, 4823-4829 (2007).

[21] N. K. Metzger, R. F. Marchington, M. Mazilu, R. L. Smith, K. Dholakia, and E. M. Wright, "Measurement of the restoring forces acting on two optically bound particles from normal mode correlations," Phys. Rev. Lett. 98, 068102 (2007).

[22] M. Guillon, O. Moine, and B. Stout, "Longitudinal optical binding of high optical contrast microdroplets in air," Phys. Rev. Lett. 96, 143902 (2007).

[23] P. W. Milonni and A. Smith, "Van der Waals dispersion forces in electromagnetic fields," Phys. Rev. A 53, 3484-3489 (1996).

[24] D. S. Bradshaw and D. L. Andrews, "Optically induced forces and torques: Interactions between nanoparticles in a laser beam," Phys. Rev. A 72, 033816 (2005).

[25] G. D. Scholes and D. L. Andrews, "Damping and higher multipole effects in the quantum electrodynamical model for electronic energy transfer in the condensed phase," J. Chem. Phys. 107, 5374-5384 (1997).

[26] P. C. Chaumet and M. Nieto-Vesperinas, "Optical binding of particles with or without the presence of a flat dielectric surface," Phys. Rev. B 64, 035422 (2001). 
[27] G. Juzeliūnas and D. L. Andrews, "Quantum electrodynamics of resonance energy transfer," Adv. Chem. Phys. 112, 357-410 (2000).

[28] D. L. Andrews and D. S. Bradshaw, "Laser-induced forces between carbon nanotubes," Opt. Lett. 30, 783-785 (2005). 